\begin{tabular}{|c|l|}
\hline Title & A theoretical study and realization of new spin quantum cross structure devices using organic materials \\
\hline Author(s) & Kondo, Kenji; Kaiju, Hideo; Ishibashi, A kira \\
\hline Citation & $\begin{array}{l}\text { MRS Proceedings, 1198, 1198-E07-01 } \\
\text { https://doi.org/L0.1557PROC-1198-E07-01 }\end{array}$ \\
\hline Issue Date & 2010-05-10 \\
\hline Doc URL & http://hdl.handle.net/2115/57073 \\
\hline Rights & ○ 2010 Materials Research Society \\
\hline Type & article \\
\hline File Information & MRS2009Fall Published.pdf \\
\hline
\end{tabular}

Instructions for use 


\section{A Theoretical Study and Realization of New Spin Quantum Cross Structure Devices using Organic Materials}

Kenji Kondo ${ }^{1}$, Hideo Kaiju ${ }^{1,2}$, and Akira Ishibashi ${ }^{1}$

${ }^{1}$ Laboratory of Quantum Electronics, Research Institute for Electronic Science, Hokkaido University, Sapporo 001-0020, Japan.

${ }^{2}$ PRESTO, Japan Science and Technology Agency, Saitama 332-0012, Japan.

\section{ABSTRACT}

We have proposed a spin quantum cross structure (SQCS) device as a candidate beyond CMOS. The SQCS device consists of two ferromagnetic metal thin films with their edges crossed, and sandwiches a few atoms or molecules. In this work, the spin dependent transport formula has been derived for SQCS devices with collinear ferromagnetic electrodes within the framework of the Anderson Hamiltonian. Also, the calculation of the magnetoresistance (MR) ratio has been done as a function of renormalized transfer matrices including magnetostriction effects and the other effects phenomenologically. It is shown that the MR ratio can be controlled by changing the renormalized coupling constants. The MR ratio is represented by a new formula. Also, we have realized an SQCS device with Ni magnetic thin-film electrodes, sandwiching poly (3-hexylthiophene) (P3HT): 6, 6-phenyl-C61-butyric acid methyl ester (PCBM) organic molecules between both the electrodes. The current-voltage characteristics of SQCS devices were measured by a four-terminal method and agree well with the theoretical results, quantitatively.

\section{INTRODUCTION}

Spintronic devices have attracted great interest from the viewpoint of new switching devices and new memory devices. Among these devices, a molecular spintronic device is one of many promising candidates since the discovery of the magnetoresistance (MR) effect in ferromagnetic metal/organic molecules/ferromagnetic metal [1]-[4]. Recently we have proposed a spin quantum cross structure (SQCS) device, in which organic molecules are sandwiched between two ferromagnetic thin films with their edges crossed [5]-[7]. The magnetic thin films are made by the evaporation method on polyethylene naphthalate (PEN) substrates. We can make SQCS devices by using the edges of evaporated metal thin films as both the electrodes. Therefore, the area of the cross point of the SQCS device can ultimately be reduced to dimensions in the order of a few square nanometers since the area size is determined by the metal-deposition rate, which can be changed from $0.01 \mathrm{~nm} / \mathrm{s}$ to the order of $0.1 \mathrm{~nm} / \mathrm{s}$. In this paper, we have developed a formula for spin dependent transport in SQCS devices with collinear $\mathrm{Ni}$ magnetic electrodes within the framework of the Anderson Hamiltonian, and we have studied the current-voltage characteristics and the MR ratio of SQCS devices with application of the theory. Also, we have realized an SQCS device with Ni magnetic thin-film electrodes, including poly (3-hexylthiophene) (P3HT): 6, 6-phenyl C61-butyric acid methyl ester (PCBM) organic molecules between both the electrodes. It is shown that the experimental results agree very well with the theoretical ones. 


\section{THEORY AND EXPERIMENT}

We investigate the current-voltage (I-V) characteristics of SQCS devices with a molecule sandwiched between two ferromagnetic metal electrodes within the framework of the Anderson Hamiltonian. The molecule is assumed to have two energy levels. The model of the SQCS device with two-dimensional (2D) electrodes is shown in figure 1(a). The energy diagram for the SQCS device model is also shown in figure 1(b).

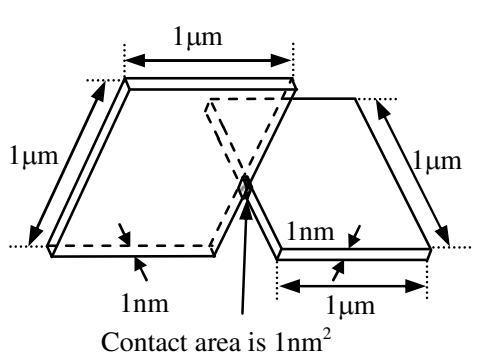

(a) SQCS model with 2D electrodes

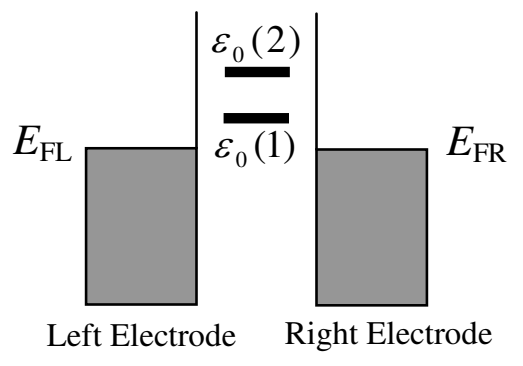

(b) Energy diagram for SQCS devices

Figure 1. (a) Schematic illustration of SQCS device model with two-dimensional (2D) electrodes. The small shaded box between the electrodes indicates the molecule position.

(b) The energy diagram for SQCS devices.

We develop the theory of spin dependent transport characteristics using the Anderson Hamiltonian. In this case, the Anderson Hamiltonian is described as follows:

$$
\begin{aligned}
& H=H_{\text {Electrodes }}+H_{\text {mole }}+H_{\mathrm{t}}, \\
& H_{\text {Electrodes }}=\sum_{\alpha=L, R} \sum_{k, \sigma} \varepsilon_{k \sigma} c_{\alpha, k \sigma}^{+} c_{\alpha, \boldsymbol{k} \sigma}, \\
& H_{\text {mole }}=\sum_{i, \sigma} \varepsilon_{0}(i) a_{i, \sigma}^{+} a_{i, \sigma}, \\
& H_{\mathrm{t}}=\sum_{\alpha=L, R} \sum_{k, \sigma} \sum_{i, \sigma}\left(V_{\alpha}^{\sigma} c_{\alpha, \boldsymbol{k} \sigma}^{+} a_{i, \sigma}+\text { H.c. }\right) .
\end{aligned}
$$

$H_{\text {Electrodes }}$ is the Hamiltonian of both the ferromagnetic metal electrodes, $\varepsilon_{k \sigma}=\frac{\hbar^{2} \boldsymbol{k}^{2}}{2 m}, m$ is the free electron mass, and $\hbar$ is Planck's constant $h$ divided by $2 \pi$. The wave vector $\boldsymbol{k}$ is a 2D vector in 2D electrodes. $c_{\alpha, k \sigma}^{+}$and $c_{\alpha, k \sigma}$ are creation and annihilation operators for electrons of wave vector $\boldsymbol{k}$ and spin index $\sigma$ in $\alpha$ electrode. $\alpha$ indicates the left or right electrode. The creation and annihilation operators obey the standard fermion anticommunication rules. $H_{\text {mole }}$ is the Hamiltonian of a molecule sandwiched between both the electrodes, and $\varepsilon_{0}(i)$ represents the $i$-th energy level of eigenstates of the molecule as shown in figure 1(b). We assumed the molecule had only two energy levels, $\varepsilon_{0}(1)=0.95 \mathrm{eV}, \varepsilon_{0}(2)=1.95 \mathrm{eV}$, estimated from Fermi levels $E_{\mathrm{FL}}, E_{\mathrm{FR}}$ of each electrode, imagining that the sandwiched molecule consists of a P3HT: 
PCBM blend. The Fermi levels $E_{\mathrm{FL}}$ and $E_{\mathrm{FR}}$ of each electrode were assumed to be equal and we used the value of $9.071 \mathrm{eV}$ for the Ni Fermi level [8]. The value of $260 \mathrm{meV}$ was used for the exchange splitting of $\mathrm{Ni} . a_{i, \sigma}^{+}$and $a_{i, \sigma}$ are creation and annihilation operators for electrons of spin index $\sigma$ in the $i$-th energy level. These operators also obey the standard fermion anticommunication rules like $c_{\alpha, k \sigma}^{+}, c_{\alpha, k \sigma} . H_{\mathrm{t}}$ is the transfer Hamiltonian between the sandwiched molecule and each electrode, $V_{\alpha}^{\sigma}$ is the transfer matrix between the molecule and electrons of spin index $\sigma$ in $\alpha$ electrode. This value determines the coupling strength between each electrode and the molecule. Considering $H_{\mathrm{t}}$ as a perturbation, we have investigated the $I-V$ characteristics from right to left electrode using the many-body perturbation technique. After tedious manipulation, the current $I$ from right to left electrode is as follows:

$$
\begin{aligned}
I_{\uparrow \uparrow}=\frac{e}{h} \int_{E_{F R}}^{E_{F R}+e V} d \varepsilon \sum_{i} & \left(\frac{4 \Gamma_{\mathrm{L}}^{+}(\varepsilon) \Gamma_{\mathrm{R}}^{+}(\varepsilon)}{\left[\varepsilon-\varepsilon_{0}(i)\right]^{2}+\left[\Gamma^{++}(\varepsilon)\right]^{2}}+\frac{4 \Gamma_{\mathrm{L}}^{-}(\varepsilon) \Gamma_{\mathrm{R}}^{-}(\varepsilon)}{\left[\varepsilon-\varepsilon_{0}(i)\right]^{2}+\left[\Gamma^{--}(\varepsilon)\right]^{2}}\right) \\
\times & {\left[f\left(\varepsilon-e V-E_{F R}\right)-f\left(\varepsilon-E_{F R}\right)\right], } \\
I_{\uparrow \downarrow}=\frac{e}{h} \int_{E_{F R}}^{E_{F R}+e V} d \varepsilon \sum_{i} & \left(\frac{4 \Gamma_{\mathrm{L}}^{+}(\varepsilon) \Gamma_{\mathrm{R}}^{-}(\varepsilon)}{\left[\varepsilon-\varepsilon_{0}(i)\right]^{2}+\left[\Gamma^{+-}(\varepsilon)\right]^{2}}+\frac{4 \Gamma_{\mathrm{L}}^{-}(\varepsilon) \Gamma_{\mathrm{R}}^{+}(\varepsilon)}{\left[\varepsilon-\varepsilon_{0}(i)\right]^{2}+\left[\Gamma^{-+}(\varepsilon)\right]^{2}}\right) \\
& \times\left[f\left(\varepsilon-e V-E_{F R}\right)-f\left(\varepsilon-E_{F R}\right)\right],
\end{aligned}
$$

where $I_{\uparrow \uparrow}\left(I_{\uparrow \downarrow}\right)$ is the current flowing from right to left electrode when two Ni magnetic electrodes have parallel (antiparallel) magnetic moments, and

$$
\begin{array}{ll}
\Gamma^{++}(\varepsilon)=\Gamma_{\mathrm{L}}^{+}(\varepsilon)+\Gamma_{\mathrm{R}}^{+}(\varepsilon), & \Gamma^{+-}(\varepsilon)=\Gamma_{\mathrm{L}}^{+}(\varepsilon)+\Gamma_{\mathrm{R}}^{-}(\varepsilon), \\
\Gamma^{-+}(\varepsilon)=\Gamma_{\mathrm{L}}^{-}(\varepsilon)+\Gamma_{\mathrm{R}}^{+}(\varepsilon), & \Gamma^{--}(\varepsilon)=\Gamma_{\mathrm{L}}^{-}(\varepsilon)+\Gamma_{\mathrm{R}}^{-}(\varepsilon) .
\end{array}
$$

Here, $\Gamma_{\mathrm{L}}^{+}(\varepsilon)$ and $\Gamma_{\mathrm{R}}^{+}(\varepsilon)\left(\Gamma_{\mathrm{L}}^{-}(\varepsilon)\right.$ and $\left.\Gamma_{\mathrm{R}}^{-}(\varepsilon)\right)$ are coupling strengths between the molecule and the majority (minority) spin electrons of left and right electrodes, respectively, which are given in the following:

$$
\Gamma_{\mathrm{L}}^{+(-)}(\varepsilon)=\pi D_{\mathrm{L}}^{+(-)}(\varepsilon)\left|V_{\mathrm{L}}^{+(-)}\right|^{2}, \quad \Gamma_{\mathrm{R}}^{+(-)}(\varepsilon)=\pi D_{\mathrm{R}}^{+(-)}(\varepsilon)\left|V_{\mathrm{R}}^{+(-)}\right|^{2}
$$

where $D_{\mathrm{L}}^{+}(\varepsilon)$ and $D_{\mathrm{R}}^{+}(\varepsilon)\left(D_{\mathrm{L}}^{-}(\varepsilon)\right.$ and $\left.D_{\mathrm{R}}^{-}(\varepsilon)\right)$ are densities of states of the majority (minority) spin electrons in left and right electrodes, respectively. $V_{\mathrm{L}}^{+}$and $V_{\mathrm{R}}^{+}\left(V_{\mathrm{L}}^{-}\right.$and $\left.V_{\mathrm{R}}^{-}\right)$are transfer matrices between the molecule and majority (minority) spin electrons in left and right electrodes, respectively. Here, we define the renormalized transfer matrices as follows:

$$
W_{\mathrm{L}(\mathrm{R})}^{+(-)}(\varepsilon)=D_{\mathrm{L}(\mathrm{R})}(\varepsilon)\left|V_{\mathrm{Ren} \mathrm{L}(\mathrm{R})}^{+(-)}\right|^{2}
$$


In these renormalized transfer matrices $W_{\mathrm{L}(\mathrm{R})}^{+(-)}(\varepsilon), V_{\mathrm{Ren} \mathrm{L}(\mathrm{R})}^{+(-)}$can include the spin polarization, physical disconnection induced by magnetostriction effects, and the other effects with application of magnetic field, phenomenologically. Changing these $W_{\mathrm{L}(\mathrm{R})}^{+(-)}(\varepsilon)$ values, we can obtain current-voltage $(I-V)$ characteristics, and estimate these values by comparing the theoretical $I-V$ characteristics with the experimental ones. Also, we can calculate the MR ratio in the following equation using the above developed theory:

$$
M R=\frac{G_{\uparrow \uparrow}-G_{\uparrow \downarrow}}{G_{\uparrow \uparrow}}=\left.\frac{I_{\uparrow \uparrow}-I_{\uparrow \downarrow}}{I_{\uparrow \uparrow}}\right|_{V=V_{0}},
$$

where $G_{\uparrow \uparrow}\left(G_{\uparrow \downarrow}\right)$ is the conductance of SQCS devices when two Ni electrodes have parallel (antiparallel) magnetic moments. They depend on the operating voltage. Then, the MR ratio is defined at $V_{\mathrm{o}} . V_{\mathrm{o}}$ is the operating voltage, which is $1.4 \mathrm{~V}$ in this calculation. We have realized an SQCS device with Ni thin film electrodes. The SQCS device sandwiches a little P3HT: PCBM blend. First, Ni thin films were evaporated on polyethylene naphthalate (PEN) substrates, and they have been sandwiched between two polymethyl methacrylate (PMMA) resins. Then, the edge of the PMMA/Ni/PEN/PMMA has been polished by chemical mechanical polishing methods. Finally, the P3HT: PCBM blend has been sandwiched between two sets of PMMA/Ni/PEN/PMMA. The Ni film is $16 \mathrm{~nm}$ thick. Therefore, the junction area of the SQCS device is $16 \mathrm{~nm} \times 16 \mathrm{~nm}$. The current-voltage characteristics have been measured by a fourprobe method at room temperature for the SQCS device with P3HT: PCBM blend.

\section{RESULTS AND DISCUSSION}

Figure 2(a) shows the calculated $I$ - $V$ characteristics of SQCS devices sandwiching a P3HT and a PCBM molecule with parallel magnetic moments under the weak coupling condition at room temperature. We regard the coupling as weak coupling when the energy of the coupling strength is smaller than that of the ambient temperature $26 \mathrm{meV}$. In this situation, we used the coupling strengths $\Gamma_{\mathrm{L}}^{+}(\varepsilon)$ and $\Gamma_{\mathrm{R}}^{+}(\varepsilon)$ of $1.57 \mathrm{meV}$. The results show sharp steps at the positions of the energy levels of the sandwiched molecule. Notice that these conductance peaks are sharp at room temperature. This fact indicates that SQCS devices sandwiching a P3HT and a PCBM molecule can be used as switching devices that work even at room temperature. Figure 2(b) shows the $I-V$ characteristics of SQCS devices under the strong coupling limit. The strong coupling limit means that the energy of the coupling strength is much larger than that of the ambient temperature. We used the coupling strengths $\Gamma_{\mathrm{L}}^{+}(\varepsilon)$ and $\Gamma_{\mathrm{R}}^{+}(\varepsilon)$ of $3927 \mathrm{meV}$. In this case, the energy level of the sandwiched molecule is almost continuous because of the wide broadening induced by the strong coupling with the itinerant electrons. Therefore, we expect the $I$ - $V$ characteristics to be Ohmic. As expected, we can obtain the Ohmic $I-V$ characteristics. The calculated resistance $R$ is $6.7 \mathrm{k} \Omega$ when the number of conductance channels is four. This is because two energy levels correspond to four channels, taking into consideration the spin degree. The calculated MR ratio is shown in figure 2(c). We have calculated the MR ratio with the renormalized transfer matrices changed, while imagining that SQCS devices have ferromagnetic materials with different spin polarization in both the electrodes, and that the coupling between 
both the electrodes is getting weak due to physical shrinkage induced by magnetostriction effects with application of magnetic field. Notice that we can obtain the giant MR effects by controlling the renormalized transfer matrices. The calculated curve is described very well by the function:

$$
M R=\frac{A-1}{A+1}, \quad \text { where } \quad A=\frac{W_{\mathrm{R}}^{+}}{W_{\mathrm{L}}^{-}}
$$
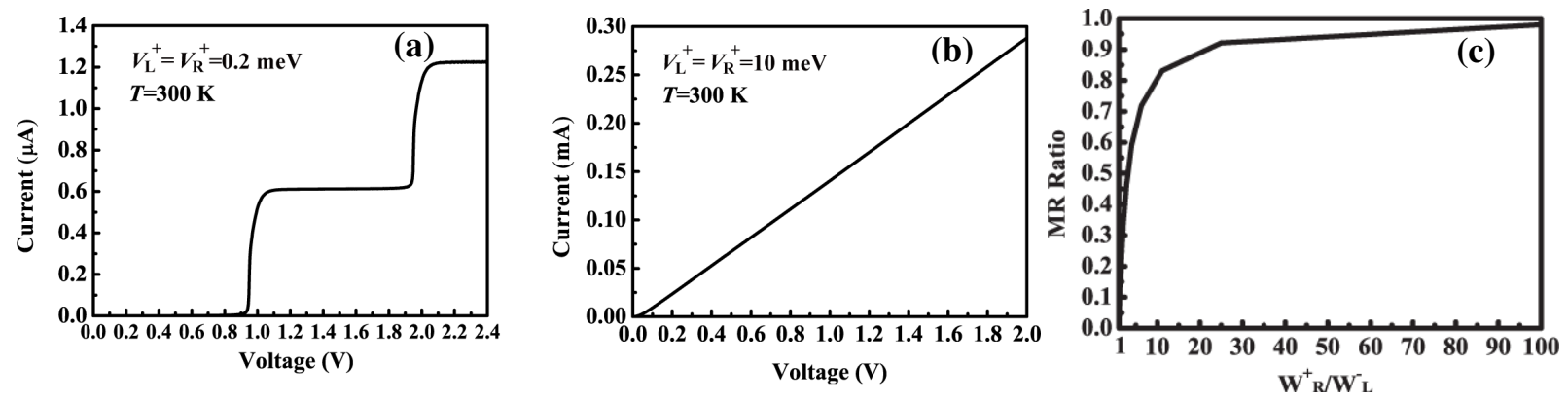

Figure 2. The current-voltage characteristics of SQCS devices sandwiching a P3HT and a PCBM molecule (a) under the weak coupling condition and (b) under the strong coupling limit. (c) The calculated MR ratio of SQCS devices.
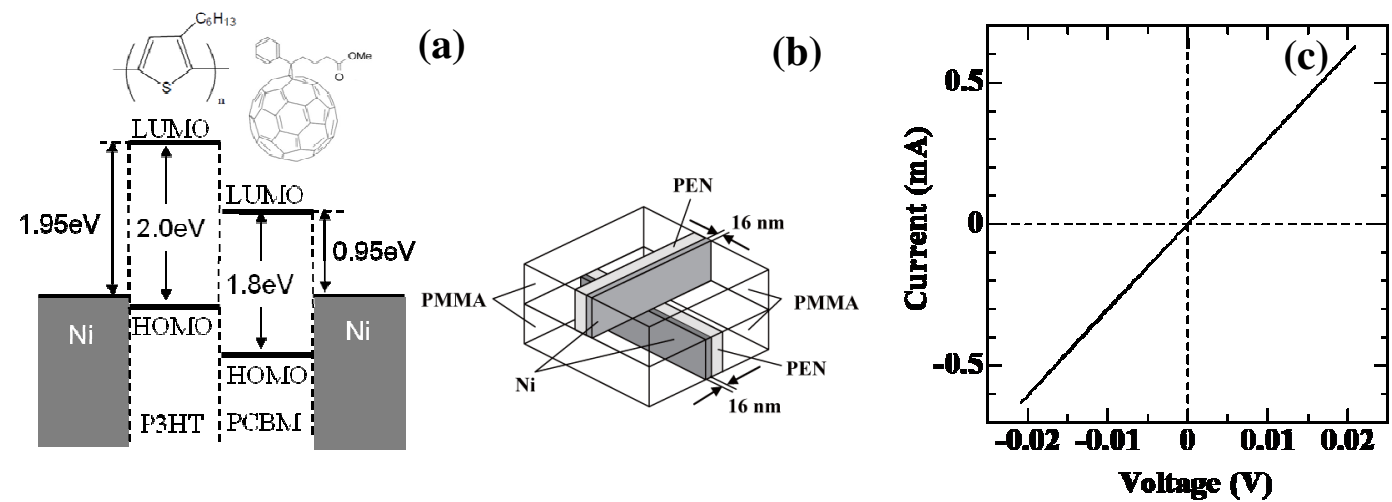

Figure 3. (a) The energy diagram for SQCS devices sandwiching a P3HT: PCBM blend [9],[10]. (b) A fabricated SQCS device. (c) Experimental $I-V$ characteristics of a fabricated SQCS device with P3HT: PCBM blend.

We find that this function is very similar to the amplitude reflection coefficient of electromagnetic waves, regarding the renormalized transfer matrix ratio as a relative refractive index. Namely, the MR ratio can be regarded as an amplitude reflection coefficient of itinerant dlike electrons from one electrode to the other electrode. Then, we have realized an SQCS device sandwiching a little P3HT: PCBM blend. Figure 3(a) shows the energy diagram for SQCS devices sandwiching a P3HT: PCBM blend, and figure 3(b) shows a schematic illustration of the fabricated SQCS device. Figure 3(c) shows the $I-V$ characteristics for the SQCS device with P3HT: PCBM blend at room temperature. You can find that the experimental $I-V$ characteristics are Ohmic. Moreover, the estimated resistance $R$ is $32 \Omega$. The diameter of a PCBM molecule is about $1 \mathrm{~nm}$. Therefore, the number of PCBM: P3HT molecules is estimated to be $256(=16 \times 16)$ 
because the junction area is $16 \mathrm{~nm} \times 16 \mathrm{~nm}$ as stated above. Here, the occupied area of P3HT was ignored since P3HT is much smaller than PCBM. Then, the number of conductance channels is 1024 (=4x256) for 256 pairs of P3HT: PCBM because a pair of P3HT: PCBM molecules have 4 channels, taking into consideration the spin degree. Then, the theoretical resistance divided by 256 is equal to $26.2 \Omega$, which is in very good agreement with the experimental result. This suggests that 256 pairs of P3HT: PCBM exist at least in the junction area, and that the developed theory is very useful for device design.

\section{CONCLUSIONS}

We have proposed an SQCS device as a candidate beyond CMOS, and calculated the $I-V$ characteristics of the devices sandwiching organic materials within the framework of the Anderson model at room temperature. It is shown that SQCS devices sandwiching organic materials can be used as switching devices that work even at room temperature. Also, the MR ratio has been calculated for SQCS devices and we can obtain the giant MR effects by controlling the renormalized transfer matrices. Then, we have fabricated an SQCS device with $\mathrm{Ni}$ electrodes. As a result, experimental $I-V$ characteristics agree very well with the theoretical results, quantitatively. Also, we have successfully estimated the number of molecules sandwiched between the electrodes. Therefore, these results suggest that SQCS devices with organic materials open up a novel research field not only for application to switching devices but also for the elucidation of the electric characterization for the small amount of organic materials.

\section{ACKNOWLEDGMENTS}

This research has been partially supported by Special Education and Research Expenses from Post-Silicon Materials and Devices Research Alliance, also by a Grant-in-Aid for Scientific Research from Japan Society for the Promotion of Science, a Grant-in-Aid for Young Scientist from MEXT, and Foundation Advanced Technology Institute.

\section{REFERENCES}

1. Z. H. Xiong, D. Wu, Z. V. Vardeny, and J. Shi, Nature 427, 821 (2004).

2. T. S. Santos, J. S. Lee, P. Migdal, I. C. Lekshmi, B. Satpati, and J. S. Moodera, Phys. Rev. Lett. 98, 0166011 (2007).

3. V. Dediu, L. E. Hueso, I. Bergenti, A. Riminucci, F. Borgatti, P. Graziosi, C. Newby, F. Casoli, M. P. De Jong, C. Taliani, and Y. Zhan, Phys. Rev. B. 78, 115203 (2008).

4. F. J. Wang, C. G. Yang, Z. V. Vardeny, and X. G. Li, Phys. Rev. B 75, 245324 (2007).

5. K. Kondo and A. Ishibashi, Jpn. J. Appl. Phys. 45, 9137 (2006).

6. H. Kaiju, A. Ono, N. Kawaguchi, and A. Ishibashi, Jpn. J. Appl. Phys. 47, 244 (2008).

7. K. Kondo, H. Kaiju, and A. Ishibashi, J. Appl. Phys. 105, 07D5221 (2009).

8. C. S. Wang and J. Callaway, Phys. Rev. B 15, 298 (1977).

9. D. E. Eastman, Phys. Rev. B 2, 1 (1970).

10. B. C. Thompson and J. M. J. Frecht, Angew. Chem. Int. Ed. 47, 58 (2008). 